\title{
Case Report of Nuchal Cord Associated Stillbirth: What to Learn From the Complaints to the Diagnosis?
}

\author{
Philippe PA ${ }^{1 *}$, Cheruiyot A ${ }^{1}$, Roselinda Nganga ${ }^{1}$, Orang'o E. Omenge ${ }^{1}$ \\ ${ }^{1}$ Department of Reproductive Health, Moi University School of Medicine, P.O Box 4606-30100 Eldoret, Kenya.
}

*Corresponding author: Philippe Amubwuomombe Poli, MD, Department of Reproductive Health, Moi University, School of Medicine, P.O Box 4606-30100 Eldoret, Kenya.

Received Date: June 21, 2019; Accepted Date: July 14, 2020; Published Date: July $20,2020$.

Citation: Philippe PA, Cheruiyot A, Nganga R, Orang'o E. Omenge. (2020) Case Report of Nuchal Cord Associated Stillbirth: What to Learn From the complaints to the Diagnosis: Observational study. Obstetrics Genecology and Reproductive Sciences, 4(1):DOI:10.31579/2578-8965/041

Copyright: ( ) 2020. Philippe Amubwuomombe Poli. This is an open-access article distributed under the terms of the Creative Commons Attribution License, which permits unrestricted use, distribution, and reproduction in any medium, provided the original author and source are credited.

\begin{abstract}
:
Nuchal cord is the umbilical cord accident least associated with stillbirths. It is a common occurrence; however, the expertise to diagnose multiple and tight loops on ultrasound is minimal, especially in limited-resource settings. We report a 30 year-old gravida 1 , who presented at a gestation of $39 / 40+6 / 7$ with a 2 day history of inability to appreciate foetal movements. An urgent obstetric ultrasound revealed absent foetal cardiac activity, reduced amniotic fluid and normal placenta but the report had no comments on the umbilical cord. She delivered a macerated male infant with a tight cord around the neck thrice. This case report highlights the importance of third trimester sonography screening of umbilical cord abnormalities and the mechanism of intrauterine foetal death associated with umbilical cord accidents.
\end{abstract}

Keywords: cord accidents, nuchal cord, stillbirth, perinatal

Abbreviations: UCA: Umbilical cord accidents; CRL: crown-rump length

\section{Introduction}

Umbilical cord accidents (UCA) are a significant cause of perinatal deaths, accounting for $10-15 \%$ of all stillbirths $[1,2]$. Cord accidents are characterized by obstruction of foetal blood flow through the umbilical cord. They include cord torsion, cord compression, cord entanglement, cord presentation, cord prolapse, cord rupture, and nuchal cord. It is important to emphasize that, almost all umbilical cord accidents are related to the abnormal length of the umbilical cord rather than its anatomical insertion into the placenta or structural abnormalities [3]. These accidents can occur antepartum or intrapartum and can influence the perinatal outcome.

Although screening and surveillance methods have been widely used for timely diagnosis and prompt appropriate management of umbilical cord accidents, a number of stillbirths associated with acute cord complications still predominantly occur in resource-limited settings. This case report emphasizes what to learn, from the patient complaints to the diagnosis in order to reduce perinatal mortality.

\section{Case report}

A 30 year-old gravida 1, presented with 2 days history of inability to appreciate foetal movements at a gestation of 39/40+6/7. She had attended her first antenatal clinic (ANC) at 9 weeks' gestation and had a total of 6 antenatal visits up to 37 weeks' gestation. All antenatal visits were uneventful. In addition, her antenatal profile was as follows: blood groupO Rhesus positive; screening for syphilis, HIV and hepatitis B were unremarkable; RBS - 4.6mmol/1; and Hemoglobin - 14.5g/dL. She received iron supplements and two Tetanus toxoid injections. The medical history was unremarkable.
Ultrasound scan done during first visit corresponded with dates and the second at 20 weeks gestation which revealed a normal pregnancy with no gross foetal anomaly seen. She presented to hospital a day before her estimated date of delivery (EDD) with a 2 day history of inability to appreciate foetal movements. An urgent obstetric ultrasound revealed absent foetal cardiac activity with reduced amniotic fluid, but normal placenta. There was no documentation related to the umbilical cord abnormalities.

Counselling was done regarding both ultrasound findings and mode of delivery. On pelvic assessment, she was found to have a Bishop's score of 2 and cervical ripening was done using misoprostol as per the Moi Teaching and Referral Hospital (MTRH) protocol. She received 5 doses of the drug at a 4 hour frequency with minimal change of the cervix over 24 hours. She was exhausted and distressed. A decision to deliver her by caesarean section was made. She delivered a macerated male infant with a tight cord around the neck thrice. There was minimal meconium-stained liquor and the foetal weight was $3200 \mathrm{gms}$. Macroscopically, the placenta was grossly normal and the umbilical cord length was $86 \mathrm{~cm}$. Patient did well post-operatively and was discharged home.

\section{Discussion}

A nuchal cord refers to an umbilical cord accident whereby the cord wraps around the foetus' neck. This can occur during pregnancy, labor or birth. It is relatively common, with prevalence rates of $6 \%-37 \%$ [1]. Due to the fact that most of these babies are born healthy, it may be tempting to dismiss nuchal cords as no cause for concern. This has no place in the modern practice of obstetrics where reducing the risk of adverse perinatal outcome is the gold standard of obstetrics care. To achieve this goal, there are three ways of appreciating the severity of nuchal cords. The first is based on the number of times the cord is wrapped around the fetal neck: 
single nuchal cord, double nuchal cord, thrice, etc,[4]. Second, whether it is wrapped loosely or tightly [4]. A "tight" nuchal cord is defined as one that cannot be slipped over the head during delivery, and may compress the foetal neck, with effects similar to strangulation [4,5]. This is associated with perinatal morbidity and mortality during labour and delivery. The third classifies the nuchal cord as type A or type B. A type A nuchal cord is "unlocked"; the umbilical end goes under the placental end, and fetal movement can easily undo it [4]. A Type B nuchal cord is "locked"; the placental end of the cord goes under the umbilical end of the cord, potentially leading to another type of umbilical cord complication known as a "true knot." This will not likely be resolved by fetal movement [4]. Clear documentation of these findings regarding the nuchal cord is strongly recommended for both quality of care improvement and legal implications. The image below shows clearly that the case report had a nuchal cord wrapped thrice, tight and unlocked (type A)

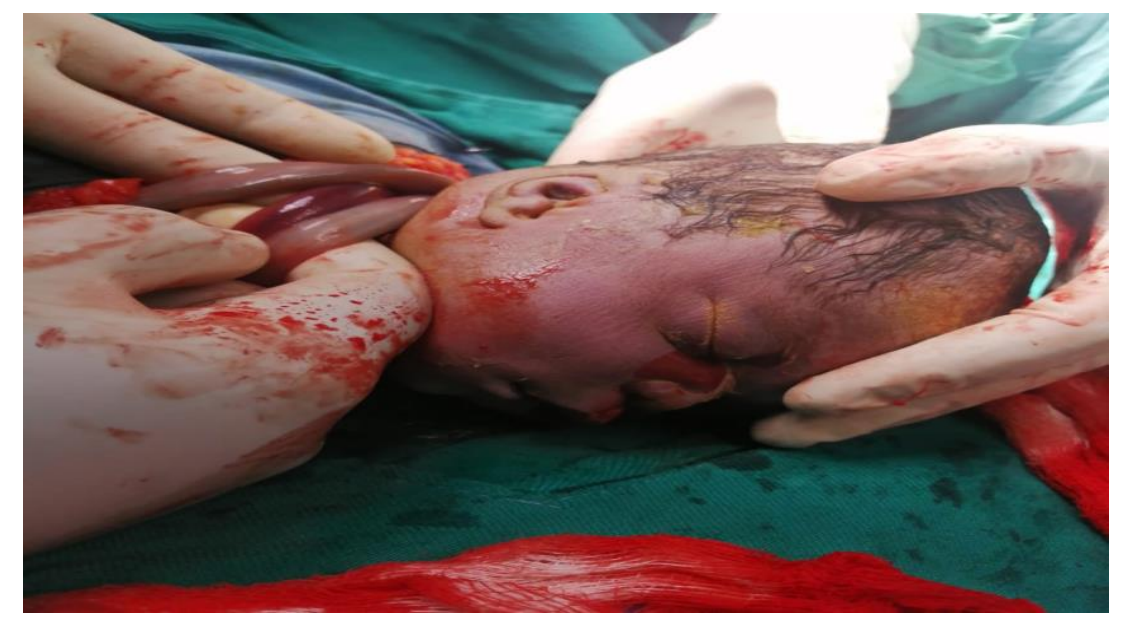

Thrice nuchal cord, tight, type A. [mage from Prof.Orang'o E. Omnge], April 2020

The incidence of nuchal cord increases with the gestational age. Larson JD and colleagues, cited by Peesay reported the overall incidence of nuchal cords to be $6 \%$ at 20 weeks gestational age and $29 \%$ at 42 weeks of gestation [6]. In addition, the occurrence of two or more loops of nuchal cords is estimated to affect between $2.4 \%$ to $8.3 \%$ of all pregnancies [6], and perinatal morbidity and mortality vary according to the severity and comorbidity described below.

Umbilical cord is considered as a lifeline of fetus. It is a multidifferentiated organ, which, embryologically originates from the foetus (allantoic core domain), between four- and six-weeks' gestation [3]. By 12 weeks of gestation, the umbilical cord is shorter than the crown-rump length (CRL); the diameter is proportional to the length and does not favour any rotation about itself [6]. Therefore, no UCA in first trimester can be possible. However, the umbilical cord characteristics such as tensile strength, diameter, weight, and length are genetically determined [6]. The current evidence has shown that these characteristics are more influenced by paternal genes and all may contribute to UCA and affect the foetus in different degrees [3-6]. For example, abnormalities of the tensile strength expose to umbilical cord rupture, which is likely to occur at the placental end [3]. The determinant factors of umbilical cord rupture include Wharton's jelly, collagen content, and muscle layer structure [3]. The rupture can only occur at breakage points and sites [36]. Regarding the umbilical cord diameter, the human umbilical cord has a reported average diameter of 1-2 $\mathrm{cm}$ and 11 helices [3]. This diameter may vary by $3 \mathrm{~cm}$ after birth and helices of as many as 380 per cord [3]. Studies have shown that various biomechanical characteristics of the umbilical cord such as elasticity, vessel wall thickness, and pressure tolerance play a major role on UCA [3-6].

With regard to the length, the occurrence of umbilical cord accidents increases as the length increases [3]. The cord is believed to elongate until as late as 36 weeks although rapid change occurs until 28 weeks, then slows [3]. The average length of umbilical cord is $55 \mathrm{~cm}$, but may ranges between $35-70 \mathrm{~cm} \mathrm{[6].} \mathrm{A} \mathrm{short} \mathrm{cord} \mathrm{is} \mathrm{defined} \mathrm{as} \mathrm{less} \mathrm{than} 35 \mathrm{~cm}$ and occurs in approximately $6 \%$ of pregnancies [6,7]. Studies have found that the short cord around the neck is more likely to wrap tightly and is most often associated with foetal inactivity, congenital malformation, intrauterine growth restriction, neurological disorders, oligohydramnios, risk of cord compression, constriction and rupture [6,7]. In addition, the presence of a short cord seems to double and triple low Apgar scores and stillbirths [6] and long-term complications characterized by learning deficiency [6]. A long cord is defined as $>80 \mathrm{~cm}$ and occurs in approximately $5 \%$ of pregnancies [7], and excessively long cord is defined by $>90 \mathrm{~cm}$. However, long umbilical cord is more associated with cord entanglement, knotting, prolapse and cord presentation, placental lesions, and cord torsion [6]. Factors that determine the umbilical cord length include the sex of foetus (male cords are longer than female cords); presentation at term (vertex fetuses may have longer lengths than term Breech fetuses with the duration of presentation unknown); parity (multigravida cord length may be longer than primigravida cord length, and the first pregnancy having a shorter length than the third); twins pregnancy (twin gestations may have fetuses with discordant lengths and shorter lengths than singletons) [3]. There is limited data about the history of long or short cords and umbilical cord accidents as predictors of subsequent UCA. However, these details are most important for the management of pregnancy.

Clinical symptoms of umbilical cord accidents are almost always similar, ranging from reduced to absent foetal movement. This has been described in previous studies as pulling sensations felt at the top of the uterus and excessive fetal movement (hyperactivity) followed by decreased foetal activity prior to fetal death [3]. According to Collins and colleagues, excessive foetal movement is a foetal response associated with the effects of umbilical cord compression [2]. It helps in repositioning the foetus and relieving the compression. However, foetal movements have been widely studied throughout pregnancy. They are reported to be predominant 
between midnight and 6 a.m. [2]. This is considered relevant for clinicians managing pregnant women to assess foetal movements over 24 hours. Better understanding of the foetal movements helps to know at what particular time the mother perceived reduced foetal movements. In the last decade, Sharkey and colleagues found that UCA associated stillbirth was likely to occur between 2 a.m. and 4 a.m. [8]. According to them, this is due to the effect of melatonin on uterus. It stimulates uterine contractions, leading to foetal hyperactivity. Studies have shown that melatonin secretion from the pineal gland begins around 10 p.m. and peaks to $60 \mathrm{pg}$ at $3 \mathrm{a} . \mathrm{m}$. as serum levels decline to below $10 \mathrm{pg}$ by 6 a.m. [8]. Heazell et al, attributes stillbirth occurrence at night to maternal supine sleeping position in the third trimester [9]. It is also possible that an acute decrease of placental perfusion following vena cava compression by a gravid uterus can stimulate the foetus to hyperactivity. For foetuses with a long umbilical cord, this hyperactivity may compromise them, especially those experiencing intermittent umbilical cord compression, nuchal cord, knotting, entanglement, rupture, exposing to decreased foetal movements therefore stillbirth.

Foetal movements are the key determinant of its wellbeing. However, many factors including amniotic fluid volume, placental location, foetal presentation, and foetal gender can affect the appreciation of foetal movements by the mother [10]. In modern obstetrics, a complaint of reduced foetal movement should be taken as a serious matter, necessitating rigorous investigations and testing, especially in third trimester. The patient history and ultrasonography are most important and can be lifesaving, if promptly conducted. Nuchal cord is not an acute event. A systematic screening for its presence, using ultrasonography in third trimester may prevent or reduce the incidence of nuchal cord associated stillbirths. The umbilical cord evaluation with sonography includes the appearance, composition, location and size of the cord [11]. In addition, a normal cord has a single vein and 2 arteries that have a twisted, rope-like appearance. Evidence has shown that an absence of twisting often is associated with a decrease in fetal movement and a poor pregnancy prognosis [3]. The biophysical profile (BPP), Umbilical Doppler study and cardiotocography (CTG) are well established parameters of assessing foetal well-being in modern obstetrics, starting at 34 weeks in low-risk pregnant women [12]. This should be included in the education of pregnant women regarding the warning signs of UCA, mainly reduced foetal movements. We understand that in our daily practice, because nuchal cord may represent a small number of foetal deaths, the motivation to investigate this reproductive tragedy may not be seen as urgent. We also understand that such screening is time consuming and not cost-effective. In addition, evidence has shown that all these pregnancy tests have high sensitivity and low specificity, increasing the rate of caesarean delivery [12]. However, there is need of better application of existing pregnancy testing tools (Ultrasound and CTG machine) in the low-risk setting to optimize prenatal care.

\section{Conclusion}

The umbilical cord is a life-connecting organ. Abnormalities in its length can be associated with foetal life-threatening complications known as umbilical cord accidents. Nuchal cord is common; however, it is least associated with stillbirths. Reduced foetal movement is the most common symptom of umbilical cord accidents. We recommend pregnant women education regarding the significance of foetal movements. In modern obstetric practice, ultrasonography and cardiotocography are necessary pregnancy testing tools even in low-risk settings, to optimize prenatal care.

\section{Acknowledgements}

We thank the individuals who participated in the management of this patient.

\section{References}

1. Joshi, K., Saxena, R., Bhat, M., Lomrod, Y., \& Verma, K. (2017). Incidence of cord around the neck and its effects on labour and neonatal outcome. Advances in Human Biology, $7(1), 15$.

2. Collins, J. H. (2012). Umbilical cord accidents. BMC pregnancy and childbirth, 12(1), A7.

3. Collins, J. H. (2014). SILENT RISK. Xlibris Corporation.Help Center: 10 things to know about nuchal cords.

4. Peesay M. (2017). Nuchal cord and its implications. Maternal health, neonatology and perinatology, 3, 28.

5. Collins, J. H. (2014, October). Umbilical cord accidents and legal implications. In Seminars in Fetal and Neonatal Medicine (Vol. 19, No. 5, pp. 285-289). WB Saunders.

6. Algreisi, F., Brown, R., Shrim, A., Albasri, S. F., Shamarani, H., \& AlZoubiadi, A. (2016). Effect of long and short umbilical cord on perinatal outcome. Int J Reprod Contracept Obstet Gynecol, 5, 4228-31.

7. Sharkey, J. T., Cable, C., \& Olcese, J. (2010). Melatonin sensitizes human myometrial cells to oxytocin in a protein kinase $\mathrm{C} \alpha$ /extracellular-signal regulated kinase-dependent manner. The Journal of Clinical Endocrinology \& Metabolism, 95(6), 2902-2908.

8. Heazell, A. E., Li, M., Budd, J., Thompson, J. M. D., Stacey, T., Cronin, R. S., \& McCowan, L. M. E. (2018). Association between maternal sleep practices and late stillbirth-findings from a stillbirth case-control study. BJOG: An International Journal of Obstetrics \& Gynaecology, 125(2), 254-262.

9. Sheikh, M., Hantoushzadeh, S., \& Shariat, M. (2014). Maternal perception of decreased fetal movements from maternal and fetal perspectives, a cohort study. BMC pregnancy and childbirth, 14(1), 286.

10. Bosselmann, S., \& Mielke, G. (2015). Sonographic assessment of the umbilical cord. Geburtshilfe und Frauenheilkunde, 75(08), 808-818.

11. Lai, J., Nowlan, N. C., Vaidyanathan, R., Shaw, C. J., \& Lees, C. C. (2016). Fetal movements as a predictor of health. Acta obstetricia et gynecologica Scandinavica, 95(9), 968-975. 